\title{
A modified sublimation purification system using arrays of partitions
}

Hyeon-Gu Jeon, Masamitsu Inoue, Nobuyasa Hiramatsu, Musubu Ichikawa*, Yoshio Taniguchi

Department of Functional Polymer Science, Faculty of Textile Science and Technology, Shinshu University, 3-15-1 Tokita, Ueda 386-8567, Japan

*Corresponding author. Tel.: +81 26821 5498; fax: +81 268215413

E-mail address: musubu@shinshu-u.ac.jp (M. Ichikawa)

\begin{abstract}
The authors report a new concept of sublimation purification system for the purification of organic semiconducting materials; using arrays of partitions for the efficient separation of the target material from impurities. The authors clearly demonstrate that the new system improves the purity of an electron transporting material for organic light-emitting devices (OLEDs), which was confirmed not only by measuring the purity directly but also by evaluating the performance of OLEDs with the material.
\end{abstract}

\section{Content}

During the past a few decades, significant progress has been made in the realization of organic electronic devices such as organic light-emitting diodes (OLEDs), organic thin-film transistors (OTFTs), and organic solar cells (OSCs). ${ }^{1,2}$ Particularly, OLEDs are very promising for the application in flat-panel displays and light source, and have progressed relatively rapidly toward commercialization since the pioneering 
works of Tang and Van Slyke., Among the many factors affecting the device performance, the purity of the active materials is one of the most important ones because it directly affects not only the efficiency but also the reliability of devices., Indeed, the material purity is a common matter of concern in organic electronics because impurities significantly affect the charge transport in organic materials.,8 Several purification methods have been developed such as zone refining from the melt, chromatography and in-situ filter technique, and so on. ${ }^{6,-11}$ Among them, gradient sublimation technique is the most useful and common means for purification of organic semiconductors since most of the material do not have a liquid phase at atmospheric pressure or below. ${ }^{11}$ In this method, the separation of target material from impurities is obtained with a constant temperature gradient along the heated long tube. The target material grows on the inside wall of the tube in a short distance from the heater with or without inert gas stream, while more volatile impurities are evacuated or grow far away from the heater, and nonvolatile impurities usually remain in the source boat or near the heating zone. However, this technique frequently requires a couple of sublimations again and again to get sufficient purity, and consequently it consumes a lot of time and resource inefficiently. The improvement of the efficiency of separating target material from impurities is urgently needed for the realization of low-cost and high-performance organic electronic devices. In this paper, we report a new concept of sublimation purification system for the efficient separation of the target material from impurities; installing arrays of partitions in the sublimation tube.

Figure 1 shows the new sublimation purification system schematically and photographs of the tube and the system. The 5 independent heating-bands control the temperature of the glass tube sectionally for the segregation of the deposition positions 
of target materials and impurities. Two arrays of partitions made of glass were installed inside of the $110 \mathrm{~cm}$ long pyrex tube; shorter one is near the inert gas inlet and the longer one is in the place of material deposition. This array of partitions promotes the separated deposition of target materials from impurities. The source boat was located at $15 \mathrm{~cm}$ far from gas inlet between two arrays of partitions. The Ar gas flow was controlled from $30 \mathrm{ml} / \mathrm{min}$ to $400 \mathrm{ml} / \mathrm{min}$, and the pressure from $80 \mathrm{~Pa}$ to $0.1 \mathrm{MPa}$. With this new system, we have tried to purify some organic materials such as tris(8hydroxyquinolinato) aluminum(III) (Alq ${ }_{3}$ ) and 1,3-bis[2-(2,2'-bipyridine-6yl)-1,2,4oxadiazol-5yl]benzene (Bpy-OXD), and compared with the result of the conventional method without the arrays of partitions.

In the conventional method, the purified materials are deposited on the inside wall of the tube rather broadly. For example, the deposition range of the purified $\mathrm{Alq}_{3}$ was from $10 \mathrm{~cm}$ to $30 \mathrm{~cm}$ depends on the pressure and the gas flow, and some other materials seem to be impurities were deposited about $10 \mathrm{~cm}$ far or even continuously from the purified $\mathrm{Alq}_{3}$ region. In the new method, the deposition range was from $5 \mathrm{~cm}$ to $15 \mathrm{~cm}$ for $\mathrm{Alq}_{3}$ mainly depends on the gas flow; larger the gas flow broader the deposition range. We could not get any distinct relation between the deposition range and the pressure. However, there is no distinct difference in final yield after purification between the two purification methods. We are able to get about $70 \%$ materials after one cycle purification from about $1 \mathrm{~g}$ source materials from both of the methods. The purified materials are deposited on the inside wall of the tube and on the array of partition simultaneously in the new method; from $30 \%$ to $50 \%$ of the purified materials were deposited on the inside wall of the tube after the sublimation. Although the new purification system could not enhance the final yield, it clearly improved the purity of 
the purified materials as below described.

One of the results for Bpy-OXD was shown in Figure 2 and Table I. Figure 2 shows the differential scanning calorimetry (DSC, Seiko Instruments, DSC6200) curves of three kinds of Bpy-OXD; raw material without further purification purchased from Hodogaya Chemicals, purified one by the conventional purification method without the array of partitions, and purified one by the new purification method. From the DSC data, we have calculated the purity of target materials using the simplified van't Hoff equation $^{12}$ expressed as follows:

$$
T_{0}-T=\frac{R T_{0}^{2} x}{\Delta H_{f}} \times \frac{1}{F}
$$

where $T_{0}$ is the melting point of the pure material, $T$ is the temperature of the sample during melting, $R$ is the gas constant, $\Delta H_{f}$ is the enthalpy of fusion of the pure material, $X$ is the mole fraction of impurity, and $F$ is the liquid fraction at $T$. The calculation process was explained elsewhere. ${ }^{12}$ The calculated purity data were summarized in Table I. For the comparison, we have also checked the purity by highperformance liquid chromatography (HPLC) ${ }^{13}$, which were shown in Table I together. From both of data, it is clear that the new system improve the purity of Bpy-OXD. Although the difference of purity seems to be very slight, the difference of EL properties of those materials was very distinct.

Figure 3 shows the luminance-voltage curves of EL cells made of two kinds of Bpy-OXD as the electron transport layer. From those curves, one can easily grasp that Bpy-OXD purified by the new system has much better electron transporting property than that purified by the conventional method. The turn-on voltage was lowered about 1 $\mathrm{V}$ to get a luminance of $1000 \mathrm{~cd} / \mathrm{m}^{2}$ by using Bpy-OXD purified by the new system; 
from $4.7 \mathrm{~V}$ to $3.9 \mathrm{~V}$. Because impurities usually play charge traps or interrupt charge transporting, the enhancement of material purity by using the new system resulted in this large enhancement of luminance in EL cell. Note that the luminance efficiency of both devices are almost the same of about $11 \mathrm{~cd} / \mathrm{A}$.

We have also checked the lifetime of the cells, and Figure 3 (b) shows the lumina nce changes under a constant current density of $75 \mathrm{~mA} / \mathrm{cm}^{2}$, where we chose a high c urrent density to accelerate the test due to decreasing influences of extrinsic factors like defects of sealing. The half-life time was increased about 1.5 times (from 50 hrs to 79 hrs). Note that the test devices were encapsulated with a fresh desiccant in a highly inert glove box (dew point of below $-50^{\circ} \mathrm{C}$ and $\mathrm{O}_{2}$ concentration of $5 \mathrm{ppm}$ ). This enhancement can be explained by the lowering of the driving voltage comes from the purity enhancement of Bpy-OXD. From these results, we can conclude that the new purification system was improved sufficiently to enhance the device performance largely by improving the purity of materials.

In summary, we developed a new sublimation purification system using arrays of partitions in the tube. Although the final yield was almost the same with that of the conventional method, the purity of material was improved as largely as the turn-on voltage of EL device was lowered about $1 \mathrm{~V}$ and the lifetime was increased about 1.5 times.

This work was supported by the Cooperative Link for Unique Science and Technology for Economy Revitalization (CLUSTER) and CLUSTER (the $2^{\text {nd }}$ stage) of the Japan’s Ministry of Education, Culture, Sports, Science and Technology. 
References

[1] S. R. Forrest, Nature 428 (2004) 911.

[2] J. R. Sheats, J. Mater. Res. 19 (2004) 1974.

[3] C. W. Tang, S. A. Van Slyke, Appl. Phys. Lett. 51 (1987) 913.

[4] S. R. Forrest, Org. Elect. 4 (2003) 45.

[5] B. Geffroy, P. le Roy, C. Prat, Polym. Int. 55 (2006) 572.

[6] D. A. Pardo, N. Peyghambarian, G. E. Jabbour, Jpn. J. Appl. Phys. 40 (2001) 4922.

[7] O. D. Jurchescu, J. Baas, T. T. M. Palstra, Appl. Phys. Lett. 84 (2004) 3061.

[8] H. J. Wagner, R. O. Loutfy, C. -K. Hsiao, J. Mater. Sci. 17 (1982) 2781.

[9] W. Warta, R. Stehle, N. Karl, Appl. Phys. A 36 (1985) 163.

[10] C. -Y. Liu, A. J. Bard, Chem. Mater. 12 (2000) 2353.

[11] S. R. Forrest, Chem. Rev. 97 (1997) 1793.

[12] K. Yamamoto, M. Momota, H. Kitamura, K. Narita, Anal. Sci. 12 (1996) 893.

[13] The experimental condition of HPLC is as follows; Column: Inertsil ODS-SP (5 $\mu \mathrm{m}, 4.6 \times 250 \mathrm{~mm}$ ), Carrier: $\mathrm{CH}_{3} \mathrm{CN}: 0.05 \%$ TFAaq $=35$ : 65, Rate: $1 \mathrm{ml} / \mathrm{min}$, Temp.: $40^{\circ} \mathrm{C}$, Det.: $254 \mathrm{~nm}$ 
TABLE I. Summary of DSC and purity data of three kinds of Bpy-OXD.

\begin{tabular}{lccc}
\hline & $\operatorname{Tm}\left({ }^{\circ} \mathrm{C}\right)$ & \multicolumn{2}{c}{ Purity } \\
\cline { 4 - 4 } & & from HPLC (\%) & from DSC (mol\%) \\
\hline w/o Purification & 264.3 & 99.04 & 98.4 \\
Conventional Method & 266.8 & 99.37 & 99.7 \\
New Method & 267.4 & 99.73 & 99.9 \\
\hline
\end{tabular}




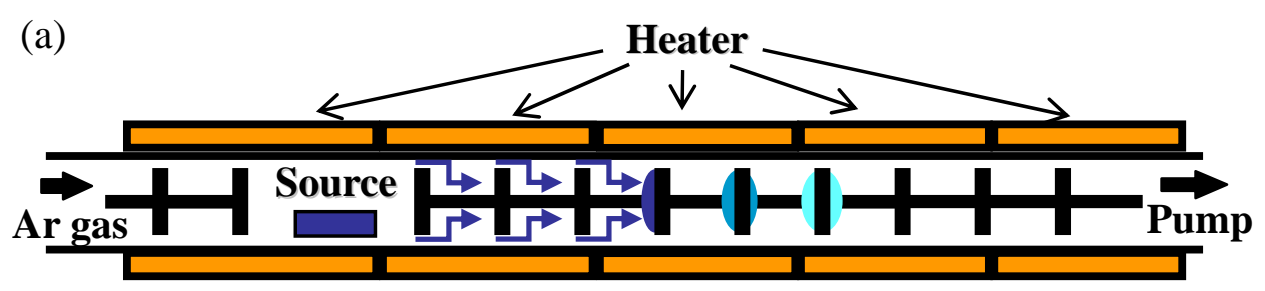

(b)

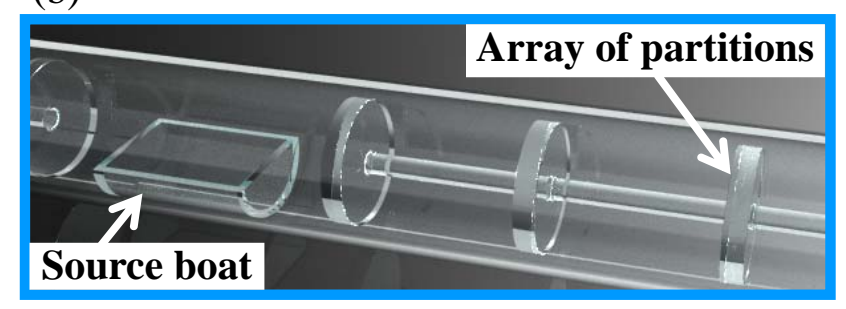

(c)

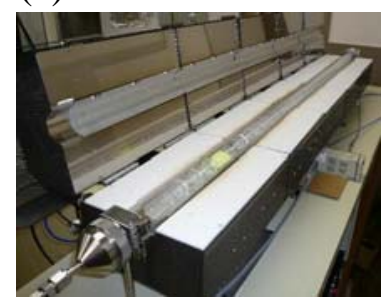

FIG. 1. (a) A schematic diagram of the new sublimation purification system where arrays of partitions is installed inside of the tube, and photographs of (b) the tube and (c) the new purification system. 


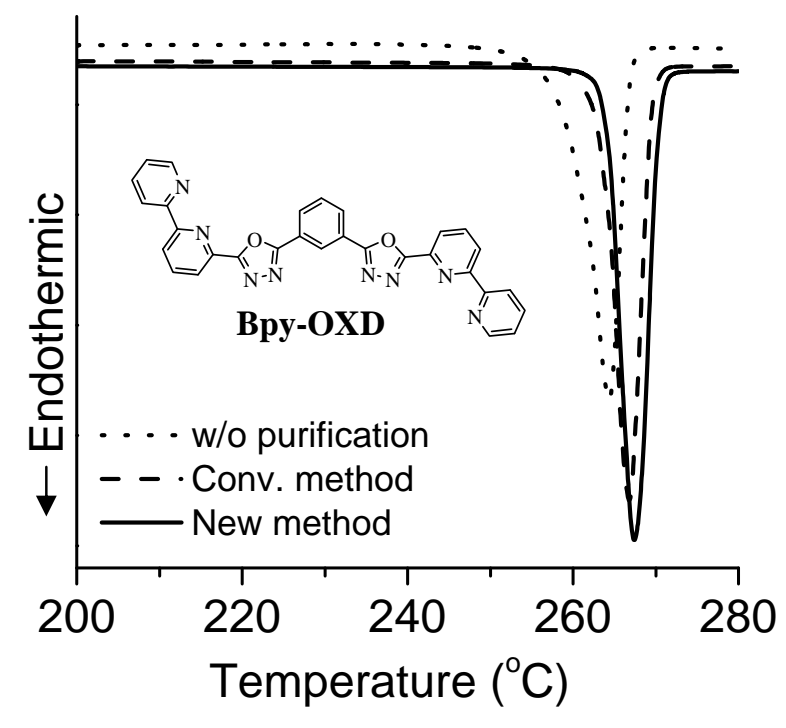

FIG. 2. Chemical structure of Bpy-OXD, and DSC curves of Bpy-OXD before (dot) and after purification by the conventional method (dash) and by the new method (solid). 
(a)

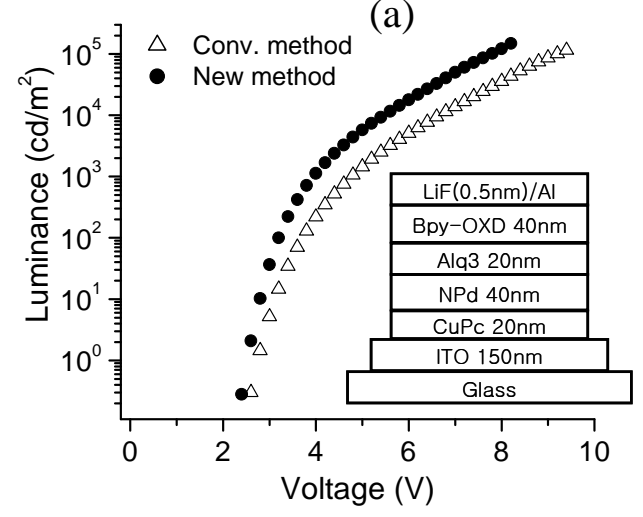

(b)

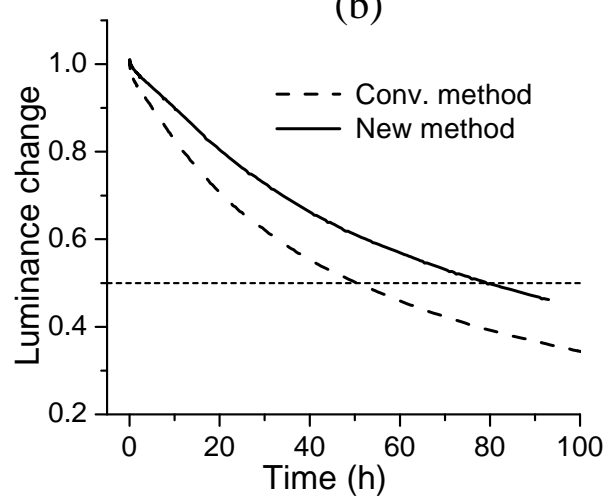

FIG. 3. (a) Luminance-voltage and (b) lifetime curves under $75 \mathrm{~mA} / \mathrm{cm}^{2}$ of EL cells made of two kinds of Bpy-OXD; purified by the conventional method ( $\triangle$, dash) and by the new method ( $\mathbf{O}$, solid). The inset in (a) shows EL cell structure used. 